$\begin{array}{cl}\begin{array}{c}\text { Revue } \\ \text { de } / \text { histoire } \\ \text { des religions }\end{array} & \text { Revue de l'histoire des religions } \\ & \begin{array}{l}1 \mid 2008 \\ \text { Varia }\end{array}\end{array}$

Orphée, sorcier ou mage?

Orpheus, sorcerer or magus?

\title{
Fabienne Jourdan
}

\section{(2) OpenEdition \\ Journals}

Édition électronique

URL : http://journals.openedition.org/rhr/5773

DOI : 10.4000/rhr.5773

ISSN : 2105-2573

Éditeur

Armand Colin

Édition imprimée

Date de publication : 1 mars 2008

Pagination : 5-36

ISBN : 978-2200-92443-0

ISSN : 0035-1423

Référence électronique

Fabienne Jourdan, «Orphée, sorcier ou mage ? », Revue de l'histoire des religions [En ligne], 1 | 2008, mis en ligne le 01 mars 2011, consulté le 19 avril 2019. URL : http://journals.openedition.org/rhr/5773 ; DOI : 10.4000/rhr.5773 
FABIENNE JOURDAN

Université Paris I - Panthéon-Sorbonne

Orphée, sorcier ou mage?

La qualification de magicien attribuée à Orphée est négative dans la période classique et les textes qui restent attachés à l'esprit de ce temps. Souvent associée au rappel de la nationalité thrace et donc barbare, elle semble cependant devenir source d'éloges dans certains textes à partir du II siècle après J.-C. La condamnation, d'une part, s'appuie sur le souvenir de la célèbre critique de Platon ou sur un jugement populaire défavorable à Orphée. L'éloge d'autre part, dû entre autres à un changement dans la manière de concevoir la philosophie, suggère de convoquer ce dernier dans la défense des "mages». Dans ce contexte, Orphée acquiert le titre, lui confère en retour ses lettres de noblesse et voit par répercussion réhabilitée sa propre figure de magicien.

\section{Orpheus, sorcerer or magus?}

The description of Orpheus as a magician was pejorative in the classical time and also in the texts which reflect the spirit of this period. However, when associated with the mention of his Thracian, and hence Barbarian origin, in some texts of the second century A.D onwards, the same criticism seems to become a source of praise. The pejorative position is on the one hand linked to Plato's famous declaration as well as to a popular opinion unfavourable to Orpheus. The praise on the other hand is due to, among other things, a new way to conceive of philosophy and suggested by the summoning of him to the magi's defence. Within this context Orpheus is raised to the status of magus, confers a title of positive value, and is thus rehabilitated as a magician. 
Pour tout un pan de la tradition païenne, Orphée est un enchanteur. Le don de séduire ou d'animer bêtes, arbres et pierres a souvent été interprété comme un «tour de magie ${ }^{1}$. Chez Euripide, le chœur d'Alceste évoque alors « le remède que la voix d'Orphée a inscrit sur les tablettes thraces »; le coryphée du Cyclope propose à Ulysse une « incantation d'Orphée vraiment infaillible » qui permettrait au tison, destiné à brûler l'œil du monstre, de se mettre tout seul en mouvement ; Admète, enfin, regrette de ne posséder cette même voix d'Orphée qui lui permettrait de séduire Déméter ${ }^{2}$. Le chant du citharède, fixé par le temps, devient pharmakon et epôidê ${ }^{3}$ - chacun des deux termes renvoyant aux vertus médicales et incantatoires de ses formules ${ }^{4}$.

1. Sur l'association spontanée des dons musicaux d'Orphée avec la magie, voir par exemple Bernabé, 1998, p. 160-167, Casadesús, 2001, p. 75 et Hernández, 2003, 2005 et 2006 b, p. 187-239. La réputation de magicien est aussi liée à l'attribution au chantre de la fondation des mystères (Hernández, 2003, p. 59-62). Peut-être apparue dès le ve siècle avant J.-C., elle se manifeste plus tard explicitement dans les papyri magiques, dont les auteurs se réclament d'Orphée pour justifier leurs formules et pratiques (Bernabé, 1998, p. 168-169, Hernández, 2003, p. 65-66).

2. Alceste, v. 966-968, T 82 Kern, 812, 813, 919 Bernabé (sur ce texte, voir Graf, 1987, p. 94 qui propose de voir ici une allusion à un rite magique où le praticien écrit sur une tablette ce que le dieu lui dicte pendant le rituel. On peut penser aussi à un usage magique des formules attribuées à Orphée dont témoigneraient par exemple les lamelles d'or. Voir Kotansky, 1991, Bernabé, 2003, Hernández, 2003, p. 57-58, 2005, p. 379-380, 2006 b, p. 242-248) ; Cyclope, v. 646-647, T 83 Kern, 814 Bernabé (sur ce texte, Hernández, 2006 b, p. 249-258) ; Alceste, v. 357-360 (sur ce texte, voir Hernández, 2006 b, p. 257 259). À ces témoignages peut être rajouté celui de Diogène de Sinope ( $F r G H$ I, 88, fr. 7-10 Snell).

3. En raison des contraintes éditoriales, sera adoptée ici une translittération très simple du grec qui ne rendra pas compte de l'accentuation et recourra à la lettre « $\hat{\mathrm{e}}$ » pour hêta et à « ô » pour omega.

4. Sur les epôidai conçues à la fois comme formules magiques et comme remèdes, voir par exemple le célèbre texte de Platon, Charmide, $157 \mathrm{a}-\mathrm{b}$, mais aussi Homère, Odyssée, XIX, v. 450 ; « Hippocrate », De la maladie sacrée, I, 1 ; Pindare, Pythiques, IV, v. 217 ; Sophocle, Ajax, v. 582 ; Platon, République, 426 b, etc. Cf. Boyancé [1936] 1972², p. 36-38, Graf, 1996, p. 30 et n. 33, p. 220 (avec bibliographie sur le sujet), Brisson, 2000 et les éditions d'epôidai par D. R. Jordan, 1992, 2000 a et b. Le terme pharmakon renvoie lui aussi à la magie comme à l'art médical. Le chœur d'Alceste qui évoque un pharmakon d'Orphée parle précisément ensuite des pharmaka des Asclépiades (v. 971). 
Ce statut de magicien ${ }^{5}$ attribué au chantre thrace est pourtant doté d'une valeur différente selon les époques. Platon ne condamne-t-il pas les charlatans qui se réclament des livres d'Orphée pour justifier des pratiques impies ${ }^{6}$ ? L'analyse des témoignages littéraires ${ }^{7}$ relatifs à cette facette du personnage montrera comment la critique qu'ils recèlent à l'origine a parfois fait place à un éloge à partir du $\mathrm{II}^{\mathrm{e}}$ siècle après J.-C. De « sorcier », Orphée passe au rang de «mage » vénérable. Chez les philosophes et les auteurs qui se réclament de ce titre, le contexte polémique joue un rôle essentiel dans l'évolution de sa renommée comme magicien. Cette étude fera apparaître la manière dont celui-ci contribue à un incessant renouvellement de la légende ${ }^{8}$.

\section{LA FIGURE DU MAGICIEN OU SORCIER JUSQU'AU DÉBUT DE NOTRE ÈRE}

À partir de l'époque classique et chez les auteurs qui restent fidèles à la pensée de ce temps, la magie fait l'objet de méfiance. Dans ce contexte, attribuer à Orphée un tel art implique sa condamnation. Avant d'examiner le détail de ce procès chez Strabon et Pline

5. Nous employons le terme magicien de manière neutre. Nous choisissons de traduire goês, qui est le plus souvent péjoratif, par «sorcier» et de translittérer magos simplement par «mage » pour laisser résonner ses acceptions tantôt négatives tantôt positives. Nous ne reviendrons pas ici sur le terme goês, longuement étudié dans son application à Orphée dans notre thèse de doctorat à paraître (voir aussi surtout Burkert, 1962). Notre attention sera ici davantage consacrée à l'emploi du substantif magos.

6. République, 364 e 3-365 a 3. Voir aussi Lois X, 909 a 8-b 6. Si ce n'est pas directement Orphée qui est visé dans la République, mais les charlatans qui se réclament de lui, le premier est néanmoins mis sur le même plan qu'Homère et Hésiode auxquels Platon reproche de fournir de mauvais modèles de justice.

7. Pour un examen du lien entre Orphée et la magie tenant compte en outre des papyri magiques et des lamelles d'or ou de plomb, voir Hernández, 2006 b. Pour une étude de l'iconographie tardive d'Orphée magicien, voir Tülük, 1998.

8. L'évolution philosophique, qui retiendra ici notre attention, n'est cependant pas le seul facteur contribuant à transformer la réputation d'Orphée. L'apparition d'ouvrages portant sur les herbes, les pierres, les remèdes ou l'astrologie, mis sous le nom de celui-ci, dans les derniers siècles avant notre ère et dans les premiers siècles après J.-C., ont également part à ce phénomène. 
l'Ancien, il est nécessaire de préciser la valeur prise par les termes désignant le magicien dès la fin du ve siècle avant J.-C. La portée du recours polémique à ces mots au début de notre ère apparaîtra ainsi plus clairement.

\section{1) La dévalorisation du magos à l'époque classique et au début de notre ère}

Le terme générique de magos, désignant à l'origine la caste des spécialistes du sacré chez les Perses ${ }^{9}$, renvoie d'abord à une catégorie de sages. Mais dès la fin du v $v^{e}$ et le début du Iv $v^{\mathrm{e}}$ siècles avant J.-C., la notion est réévaluée négativement ${ }^{10}$. Cette évolution peut s'expliquer de deux manières. Selon un point de vue historique, d'après J.N. Bremmer, elle serait due à la venue croissante de « mages » ou professionnels des rites sacrés itinérants en Ionie et en Grèce dès la fin du ve siècle avant J.-C. ${ }^{11}$ D'un point de vue spirituel,

9. Voir Xanthos de Lydie, FGrH 765 F 31-32, Hérodote, Histoires, I, 101 (sur l'origine perse) ; VII, 7, 43, 113, 171 (sur leur rôle dans les offrandes du roi) ; I, 140 (dans les rites funéraires) ; I, 107, 120, 128, VII, 19, 37 (dans l'interprétation des rêves) ; Xénophon, Cyropédie, VIII, 3, 11; (Ps-)Platon, Alcibiade Majeur, 122 a. Malgré l'apparente neutralité de la définition, Hérodote n'est pas sans sarcasmes à l'égard des mages (voir VII, 191, contra Bremmer, 1999, p. 5). Sur les mages en général, voir par exemple Bidez-Cumont, 1938 et Bremmer, 1999 ; voir aussi les remarques de Smith, 1978, p. 71-72 et de Graf, 1996, p. 24 et n. 2, p. 218.

10. Il s'agit néanmoins seulement d'une tendance. Voir Abt, 1908, p. 107108. Pour des emplois péjoratifs de magos dès le $\mathrm{v}^{\mathrm{e}}$ siècle, voir par exemple, sinon Héraclite (DK 14) dont le témoignage est fourni tardivement par Clément d'Alexandrie, Sophocle, Edipe roi, v. 387, Euripide, Oreste, v. 1497, Eschine, III, 137, Ps.-Hippocrate, Sur la maladie sacrée, 2, 3, 4, 18. Sur ces textes, Bremmer, 1999, p. 3-6 qui tend à distinguer entre une vision favorable aux magoi, commune aux historiens et philosophes aristotéliciens, et une appréciation négative chez les dramaturges, orateurs et philosophes. Cette distinction est néanmoins à nuancer dans la mesure où le témoignage d'Hérodote n'est peut-être pas si neutre qu'il n'apparaît (voir la note ci-dessus) et où nous ne disposons que de fragments tardifs des philosophes aristotéliciens concernés.

11. J. N. Bremmer (1999, p. 7-9) appuie son hypothèse sur le témoignage fourni par la colonne VI du Papyrus de Derveni où seraient selon lui évoqués des magoi qui auraient adapté leurs traditions avestiques aux rites helléniques d'une manière destinée à déplaire aux Grecs. Cette idée n'est pas retenue par F. Casadesús (2001, p. 78), M. R. Hernández (2003, p. 612, 006 b, p. 466-467), 
ce «changement de paradigme », selon l'expression de F. Graf ${ }^{12}$, serait à lier à une évolution de la philosophie et de la médecine de l'époque. La première, depuis Héraclite et surtout chez Platon, conçoit les dieux comme des modèles de vertu et rejette par conséquent tout rite censé permettre d'acheter directement leurs faveurs. La seconde commence à séparer nettement la nature et les dieux dont elle nie l'intervention directe dans les maladies - initiative qui ruine les prétentions des guérisseurs traditionnels. À partir de ce moment, la «magie » (mageia) est alors surtout conçue comme une pratique exercée par les ennemis d'Athènes en référence aux guerres médiques. Elle désigne, de manière générale, l'art des rites, initiations, prédictions, charmes bénéfiques ou maléfiques, exercé en dehors de la religion officielle de la cité. Platon n'évoque cependant pas les magoi en ce sens, peut-être pour éviter un malentendu. Il choisit d'autres termes et préfère dénoncer les « prêtres mendiants » (agurtai), «devins » (manteis) et « sorciers » ou «charlatans » (goêtes), dont l'art n'est mentionné de manière neutre qu'exceptionnellement dans le Banquet (202 e 7-9). Lorsque le philosophe ne dresse plus seulement la liste des rites qui visent à opérer un contact entre les hommes et les dieux comme c'est le cas dans ce dialogue, mais lorsqu'il se préoccupe, dans la République ou les Lois, de leurs effets dans la cité, il condamne toutes les formes de goêteia ${ }^{13}$.

L'évolution sémantique de la notion de «magie » qui commence véritablement au $\mathrm{v}^{\mathrm{e}}$ siècle est néanmoins déjà perceptible avant Platon. Les trois exemples d'Euripide cités en introduction peuvent être relus à la lumière de cette évolution. La puissance incantatoire d'Orphée est en effet mentionnée chez le dramaturge dans des contextes qui, en réalité, en annulent toute valeur : elle est invoquée

G. Betegh (2004, p. 78) et A. Bernabé (2006), selon lesquels l'évocation des magoi dans le Papyrus de Derveni ne comporterait aucune valeur péjorative. Le caractère lacunaire de ce texte ne permet néanmoins pas de trancher définitivement la question de savoir si son auteur est favorable aux magoi qu'il mentionne ou s'il décrit plutôt leurs pratiques avec une certaine distance pour mettre en valeur la sienne comme exégète des vers d'Orphée.

12. « Paradigmenwechsel », Graf, 1996, p. 31 et plus généralement p. 26-36.

13. Graf, 1996, p. 27, Bremmer, 1999, p. 4, Casadesús, 1998, Hernández, 2003, p. 59-60. 
deux fois par des personnages lâches (Admète et le coryphée du Cyclope), qui cherchent une échappatoire pour éviter d'avoir à affronter le danger de descendre aux Enfers ou d'aveugler un ennemi terrifiant ; son efficacité est niée par le chœur d'Alceste qui a rappelé dans les vers précédents qu'il n'est en réalité aucun remède contre la Nécessité, qui, seule, est supérieure à tout (v. 965-966). Euripide ne prête donc pas non plus crédit à la voix populaire qui chercherait encore quelque expédient dans le prétendu pouvoir «magique »du chant orphique.

Ainsi, dès l'époque classique et chez tous les auteurs qui, par la suite, demeurent rigoureusement fidèles à la pensée de Platon, décrire Orphée comme un magicien, quel que soit le terme choisi (goês ou magos), recèle nécessairement une critique.

\section{2) Orphée goês, barbare mis à mort pour son imposture chez Strabon}

Le jugement négatif porté sur Orphée par le biais de la qualification de goês est explicite chez Strabon en 17 ou 18 après J.-C. ${ }^{14}$. Rappelons brièvement le projet du géographe pour bien comprendre le contexte de son réquisitoire.

Strabon décrit en dix-sept livres les régions qui entourent la Méditerranée à l'intention des gouvernants ou, du moins, des lecteurs intéressés par la géopolitique et l'administration ${ }^{15}$. Témoin de la grandeur de Rome, il unit à son chauvinisme grec son admiration pour l'Empire afin de donner l'image d'une Europe autonome, gouvernée par les «peuples dominants que sont les Grecs d'abord, puis les Macédoniens et les Romains ensuite » (II, 2, 26). Strabon demeure plein de suspicion à l'égard des indigènes qu'il évoque («peuples peu policés et sauvages », II, 5, 26) et de leurs traditions. Même s'il semble suivre le précepte d'Ératosthène qui désapprouve la distinction commune entre Grecs et Barbares inculquée à Alexandre et encourage au contraire le jeune général à n'établir de différence

14. Date donnée pour le livre VII par R. Baladié dans son édition du texte aux Belles Lettres (CUF), Paris, 1989, p. 8.

15. Sur Strabon, Honigmann, 1931 ; voir aussi l' article « Strabon» in Der Kleine Pauly, 5, 1975, col. 381-385, Sirinelli,1993, p. 213-215. 
entre les hommes qu'eu égard à la vertu (I, 4, 9), cette vertu, selon le géographe, dépend d'une éducation d'homme libre, c'est-à-dire fondée sur la culture grecque.

Dès lors, quand, dans son septième livre consacré entre autres à la partie septentrionale de l'Oikoumenê, Strabon rapporte la légende d'Orphée né au pied du mont Olympe, aux confins nord de la Grèce, il est loin d'accorder à ce Cicone (ou Thrace - il s'agit d'un autre nom du même peuple) la gloire du héros civilisateur. Il le relègue au contraire au rôle de charlatan dont les prétentions musicales et religieuses n'auraient eu que fins lucratives puis politiques. Une telle imposture expliquerait selon lui la mise à mort du goês thrace par ses compatriotes les plus lucides :

«Au pied de l'Olympe se trouve la cité de Dion. À côté il y a un village, Pimpléia. C'est là, dit-on, que vécut Orphée le Cicone : un charlatan (goês) qui commença par mendier en faisant pour cela usage de la musique et, en même temps, de la divination et célébrations de mystères; puis, s'estimant alors lui-même digne de plus grandes choses, il s'acquit une foule d'adeptes et s'arrogea du pouvoir. Cela étant, les uns l'accueillirent volontiers, mais d'autres, soupçonnant un complot et une action de force, s'unirent contre lui et le tuèrent. À côté de là, il y a aussi Libèthre. (VII, 330, fr. 18, T 40 et 84 Kern, 554, 659, 816-817, 936 et 1049 Bernabé). ${ }^{16}$ »

L'analyse du texte montrera ici comment la critique du magicien barbare par Strabon s'appuie sur celle du goês par Platon et constitue en cela un «morceau », dont la marque classique justifie peut-être son choix par l'auteur de l'anthologie qui le cite.

Strabon ne loue pas un «mage » barbare. Ses positions relatives à l'Empire, d'une part, ne l'encouragent pas à exalter une figure mythique d'origine étrangère. Ses revendications stoïciennes ${ }^{17}$, d'autre part, ne l'empêchent pas d'inscrire sa condamnation dans la suite directe de celle de Platon. Il choisit ainsi à dessein le substantif goês et l'associe au participe agurteuonta qui rappelle les «prêtres mendiants » de la République (agurtai, 364 b 5). C'est dans l'esprit de ce dialogue qu'il relate une version de la légende où l'imposteur

16. Nous traduisons nous-même tous les textes cités. Sur ce fragment, voir aussi Bernabé, 2001 et Hernández, 2003, p. 63-64.

17. Strabon répète souvent qu'il est stoïcien (I, 2, 2 ; I, 2, 34 ; II, 3, 8) et chez Stéphane de Byzance il est nommé le «philosophe stoïcien ». 
représente une menace politique ${ }^{18}$. Il dépeint Orphée à l'image des sophistes et charlatans qui tentent, par leurs paroles et promesses, de circonvenir les âmes faibles afin d'abord de gagner de l'argent puis de s'emparer du pouvoir. La manière dont Strabon rapporte ce récit relève donc moins, contrairement à ce qu'il prétend, d'une tradition locale, que d'une version critique largement marquée par le jugement de Platon et apparemment reçue par les commentateurs d'Homère auxquels il emprunte ${ }^{19}$. Ses convictions politiques incitent Strabon à réduire la valeur d'une culture de source barbare. Il les sert en puisant à une source qui utilise l'image dénonciatrice du charlatan (goês) devenue traditionnelle depuis Platon et la réécrit peut-être pour amplifier la condamnation ${ }^{20}$. Ses affinités philosophiques sont mises entre parenthèses. Elles auraient dû en effet lui interdire ce mépris d'Orphée, considéré souvent dans l'école stoïcienne comme poète dont les vers, bien interprétés, auraient annoncé la doctrine du Portique ${ }^{21}$. Mais il faut sans doute bien distinguer l'image du citharède ou du mystagogue en tant que personnage légendaire de

18. F. Graf (1987, p. 89-90) rapproche ce passage de la tradition relative au destin de Pythagore à Crotone qui, après avoir rassemblé des adeptes et acquis du pouvoir, fut assailli avec les siens dont beaucoup furent tués.

19. À savoir Démétrios de Skepis (akmè vers 150) et Apollodore d'Athènes (ca. 180-110 av. J.-C.). Le contenu des fr. 17 (qui signale qu'Orphée a vécu à Pimpléia, bourg qui dépend de la cité de Dion) et 18 remontent vraisemblablement à ces deux auteurs d'après R. Baladié (1989, p. 25 -27). Le fait que Strabon fasse d'Orphée un Cicone semble confirmer cet emprunt à un commentaire du livre II de l'Iliade puisqu'Homère parle de ce peuple dans ce passage (v. 846). L'attribution de cette nationalité semble dériver de l'épopée. Elle réapparaît chez le Ps.-Aristote, fr. 641, 48, Virgile, Géorgiques, IV, v. 520, Ovide, Métamorphoses, XI, v. 4, Souda, art. « Orphée».

20. Une version plus courte de son texte se trouve chez Eusthate, Commentaire à l'Iliade (Livre II, v. 596), § 299, 1. 7-10 (Strabon, VII, fr. 18 a Baladié): «Le Cicone Orphée commença par mendier. Ensuite, s'estimant lui-même digne de plus grandes choses, il se gagna une foule d'adeptes et en tira du pouvoir, mais fut tué à cause d'une rébellion. C'était un charlatan (goês) qui recourait à la musique, à la divination et à la célébration de mystères. » La forte ressemblance entre ce texte et le nôtre tendrait à confirmer l'idée que Strabon réécrit un passage qu'il emprunte à un commentateur d'Homère. Soit il l'amplifie, soit Eusthate le raccourcit. Quoi qu'il en soit, Strabon choisit la version la plus longue qui insiste sur le danger politique constitué par le personnage.

21. Voir par exemple Cicéron, De Natura Deorum, II, 67. 
celle du poète dont les vers importent plus que la personne. Les philosophes s'intéressent surtout au second, utilisant ses textes pour soutenir leurs propres théories. Les néo-Platoniciens fourniront le meilleur exemple de cette attitude ${ }^{22}$. Strabon quant à lui ne s'intéresse qu'au premier.

L'aspect classique de la critique véhiculée par l'épisode légendaire tel que le rapporte le géographe a sans doute incité le rédacteur de l'Épitome ${ }^{23}$ qui nous transmet indirectement cette partie du livre VII à choisir ce texte. Pour composer son anthologie, il lui fallait vraisemblablement des passages évoquant des lieux célèbres liés à des récits mythiques. Une version de la mort d'Orphée devait être bienvenue dans ce contexte. Celle présentée ici avait peut-être aussi l'avantage de constituer une interprétation critique conforme au réquisitoire de Platon : une véritable réécriture de la légende dictée par une condamnation philosophique ancestrale. Elle pouvait alors aisément constituer un « morceau ».

Résumons-nous. Strabon exprime sa méfiance à l'encontre des Barbares en réécrivant, sous l'inspiration de Platon ou des commentateurs d'Homère fidèles au philosophe, une version de la mort d'Orphée. Le citharède y fait figure de goês ayant mérité son sort en raison du danger politique qu'il représente. L'empreinte classique de la condamnation a peut-être contribué au choix de ce passage par l'auteur de l'anthologie qui nous transmet le texte du géographe. C'est elle qui, à travers les générations, inspire l'image négative d'Orphée magicien.

\section{3) Orphée, inventeur de la magie noire? (Pline l'Ancien)}

Une même condamnation aurait pu se retrouver au I ${ }^{\mathrm{er}}$ siècle après J.-C. chez Pline l'Ancien. Il apparaîtra que seuls ses scrupules d'historien interdisent au Naturaliste de la formuler explicitement.

22. Proclus par exemple ne s'intéresse jamais au citharède (sinon pour le sauver des griffes de Platon, ce qui est le plus évident dans l'exégèse du mythe d'Er, in Commentaire à la République, XVI ${ }^{\mathrm{e}}$ dissertation, §§ 313-315), mais seulement au poète qui a introduit les mystères et écrit les vers récités à cette occasion ou expliquant la doctrine qui les fonde.

23. Epitome Vaticana, in codice Vaticano gr. 482 servata, saec. XIV ${ }^{1}$. 
Dès les premiers mots du livre de l' Histoire naturelle consacré à l'histoire de la magie, Pline l'Ancien dénonce les impostures de cet art, considéré comme le plus frauduleux de tous et comme doté d'un grand pouvoir parce qu'il recèle en lui-même celui des trois autres qui exercent leur emprise sur l'esprit humain, à savoir la médecine, la religion et l'astrologie (XXX, I, 1-2). Après avoir rappelé l'origine perse communément admise de la magie (XXX, II, 3), le Naturaliste émet quelques doutes sur la tradition relative à son prétendu fondateur Zoroastre (XXX, II, 4). Avant de lui donner pour initiateur historique le mage Ostanès ${ }^{24}$ qui « infecta le monde des germes de cet art monstrueux » (XXX, III, 8), il ajoute en effet :

«je croirais que c'est Orphée le premier, qui, de proche en proche, a propagé cette superstition jusqu'aux régions voisines, porté à cela par la médecine, si la Thrace où il vivait n'avait pas tout entière ignoré la magie (XXX, III, 7, T 83 Kern, 814 Bernabé). ${ }^{25}$ »

Même s'il doit renoncer à cette idée, Pline a été tenté de faire du citharède le fondateur de la magie. Seules ses sources l'auraient empêché d'affirmer cette théorie qui l'aurait volontiers convaincu. Il est étonnant qu'il n'ait pas songé à Zalmoxis, comme le fera plus tard Apulée, pour voir dans les Thraces un peuple initié à cet art. C'est peut-être parce que les incantations de ce dieu et législateur auraient été bonnes ${ }^{26}$ qu'il ne l'assimile pas à un magus. La magie que décrit le Naturaliste est en effet ce que nous appelons familièrement la «magie noire », art « détestable, inefficace et vain » qui emprunte son apparente efficacité à la médecine, mais relève en réalité de l'art des empoisonneurs (XXX, VI, 17); qui prétend à quelque rapport direct avec les dieux, mais n'est autre que « superstition ». Le jugement porté ici sur Orphée est rendu difficile par la formulation à l'irréel du passé. Pline soupçonne apparemment le citharède d'avoir recouru à la médecine pour prétendre à des dons de « magicien », mais il semble qu'il lui est impossible de l'affirmer

24. Ostanès accompagnait Xerxès dans son expédition contre les Grecs. Sur ce personnage, voir Bidez-Cumont, 1938, Tome I, p. 167-212 et Tome II, p. 267-356.

25. Sur ce texte, voir aussi Hernández, 2003, p. 63.

26. Du moins d'après ce que Platon faire dire à Socrate dans le Charmide, 157 a $4-5$. 
parce qu'il n'a pas de preuve d'un mauvais usage des incantations (ou de l'existence de la magie noire) en Thrace. Il voudrait accuser Orphée, mais ne le peut, malgré lui. L'Histoire naturelle constituant la somme des savoirs de l'époque et des idées reçues en matière de morale, ce conflit apparent entre une conviction et des sources historiques (peut-être limitées, mais prises au sérieux) révèle que, pour Pline et ses contemporains, Orphée est un magicien au mauvais sens du terme. Ses prodiges, qui contreviennent aux lois naturelles et constituent un usage détourné de la médecine, ses prétentions religieuses, qui dissimulent mal une vaine superstition, lui valent la réputation de «sorcier ». Ainsi Pline et ses contemporains héritent, comme Strabon, de l'image du goês marquée par la critique de Platon $^{27}$.

Les exemples de Strabon et Pline l'Ancien témoignent de la fécondité du procès de Platon à l'encontre des goêtes et d'Orphée, susceptible, à travers les générations, d'inspirer de nouveaux épisodes de la légende. Le poète thrace se voit ainsi tantôt mis à mort comme « sorcier» ou charlatan représentant un danger pour la cité, tantôt soupçonné d'être le fondateur de la magie noire. Dans les deux cas, sa réputation de magicien lui vaut les plus vives incriminations. Par la magie du contexte polémique néanmoins, un tel réquisitoire se transforme parfois en apologie.

\section{LA RÉHABILITATION DE LA FIGURE D'ORPHÉE, « MAGE » THRACE, À PARTIR DU II ${ }^{\mathrm{e}}$ SIÈCLE APRÈS J.-C.}

Une autre évolution de la pensée philosophique, perceptible dès le ${ }^{\text {er }}$ siècle avant J.-C. ${ }^{28}$ mais surtout sensible, dans les textes qui nous concernent, à partir du $\mathrm{II}^{\mathrm{e}}$ siècle après J.-C., entraîne une

27. Cela n'empêche pas Pline de mentionner par ailleurs un filtre d'amour dont Orphée aurait eu le secret et d'évoquer certaines connaissances médicales qu'il aurait transmises (Histoire Naturelle, XX, 32, XXVIII, 34 et 43. Voir Hernández, 2003, p. 66). Le Naturaliste connaît la réputation de « scientifique » (sur ce terme, Hernández, 2006 a) qui devient celle d'Orphée à son époque, mais ne la juge pas encore positivement.

28. Avec Posidonius (135-40 av. J.-C.) d'après Dörrie, 1972, p. 160. 
reconsidération du statut du magicien ou plutôt cette fois du « mage », le terme magos étant privilégié dans ce contexte. Si, selon F. Graf, la notion de goêteia paraît d'abord être neutre puis se charger d'une connotation péjorative lorsqu'elle est associée à celle de mageia ${ }^{29}$, il nous semble qu'à partir du II siècle (et peut-être avant) ce rapport s'inverse : la désignation de magos parait redevenir objective ou positive, tandis que celle de goês rester dépendante de la critique de Platon. C'est l'association au mot goês qui rend magos péjoratif ${ }^{30}$. Ce nouveau « changement de paradigme » s'observe principalement dans la défense des célèbres accusés pour magie. Une fois expliqué ce qui peut le motiver dans la philosophie de l'époque et dans l'image d'Orphée qui se développe au même moment, une étude des procès pour goêteia où le poète thrace est appelé à témoin permettra de comprendre comment son personnage contribue à la réhabilitation de la figure du magos et comment, par répercussion, sa propre image de magicien est revalorisée.

\section{1) Un mage barbare au début de notre ère}

Trois facteurs liés à l'évolution des conceptions philosophiques contribuent à une réhabilitation de la qualité de «mage » au début de notre ère et participent simultanément à une revalorisation d'Orphée.

1. Le premier est sans doute la tendance des philosophes de l'époque (les platoniciens en particulier) à exalter tout type de relations directes avec les dieux, à l'instar de celles prétendument réalisées par les sages du passé comme Pythagore ou Orphée. Elle trouvera son point culminant dans le néo-platonisme.

2. Depuis la période hellénistique, c'est-à-dire depuis l'avènement d'Alexandre, elle se double d'une nouvelle estime accordée aux Barbares dont font partie les Thraces ${ }^{31}$.

29. Graf, 1996, p. 28-29 en référence au texte de Gorgias que nous avons cité.

30. Quand Méragène parle de manière neutre d'Apollonios, il cite le « mage et philosophe ». Lorsqu'il le critique, il soupçonne sa mageia de n'être en réalité qu'art de goês, voir Origène, Cels. VI, 41.

31. Sur l'évolution de la notion de barbare dans la sphère païenne, voir Jüthner, 1950, col. 1173-1175, Schwabl, H. et al., 1961, Reverdin, 1961, Opelt, Speyer, 1967, p. 251-267, Dörrie, 1972, Lévy, 1984. 
3. À ce pragmatisme politique déjà ancien s'ajoute enfin la conception stoïcienne de l'histoire du logos parmi les hommes ${ }^{32}$ selon laquelle les peuples les plus proches des origines seraient encore en possession d'une sagesse qui se serait progressivement perdue au cours des âges ${ }^{33}$. Ainsi, au $\mathrm{II}^{\mathrm{e}}$ siècle après J.-C., le Platonicien Celse, dont la pensée est fortement influencée sur ce point par celle de Posidonius ${ }^{34}$, peut déclarer que les Perses (d'où sont issus les mages) et les Odryses (peuple thrace auquel appartient aussi Orphée) comptent parmi les premiers témoins de cette sagesse divine dont ils héritent et à laquelle ils participent encore ${ }^{35}$.

À cette évolution propre à la philosophie s'en ajoute une seconde qui concerne plus spécifiquement la figure d'Orphée et fait de celuici un «scientifique », ou du moins un expert dans le domaine des plantes, des pierres, de la médecine, des remèdes et enfin de l'astro$\operatorname{logie}^{36}$. Le nom du chantre thrace est en effet utilisé comme garant d'autorité, en raison de son antiquité et de la légende qu'il véhicule, par les auteurs de traités portant sur ces sujets dont certains connaissent un véritable succès jusqu'à la Renaissance ${ }^{37}$. Il n'en demeure pas moins que, sans l'évolution des conceptions spirituelles de l'époque, cette image d'Orphée magicien pourrait continuer à éveiller le mépris des philosophes et des individus qui prétendent à ce titre.

Nous essaierons donc ici de montrer comment la transformation des cadres de pensée contribue, dans le contexte des procès pour magie, à la revalorisation de la figure d'Orphée magicien, en passant nécessairement par celle du « mage », philosophe dont les ancêtres

32. Dörrie, 1972, p. 159.

33. Idée qui fait très sérieusement affirmer à Diodore $(3,2)$ que les Éthiopiens sont pourvus des meilleures mœurs parce qu'ils ne connaissent pas la civilisation hellénique.

34. Dörrie, 1972, p. 166. Le médio-platonisme en général est très marqué par le stoïcisme dont il cherche parfois à se déprendre, mais dont il préserve néanmoins certaines idées. Posidonius, qui aurait tenté de concilier doctrines de Platon, Aristote et Zénon, est souvent considéré pour cela comme précurseur du néo-platonisme.

35. Origène, Cels. II, 14.

36. Sur cette image d'Orphée « scientifique », voir Hernández, 2003, p. 6668, 2006 a et 2006 b, p. 275-342.

37. Bernabé, 1998, p. 171. 
spirituels sont les antiques sages barbares et dont les doctrines, voire les rites, permettraient d'entrer directement en contact avec les dieux. Pausanias est peut-être le premier témoin de cette évolution dans les jugements qu'il rapporte relativement au chantre thrace.

\section{2) Orphée, le «terrible mage » chez Pausanias}

Les mentions d'Orphée chez Pausanias montrent qu'à partir du $\mathrm{II}^{\mathrm{e}}$ siècle, les qualifications du citharède comme «Thrace » et comme «mage » non seulement vont de pair, mais s'imposent.

Pausanias, qui écrit vers 160 après J.-C., semble distinguer un Orphée thrace ${ }^{38}$ qui serait un «mage », d'un Orphée grec, poète auteur de vers destinés aux initiations ${ }^{39}$. Cette opposition est particulièrement sensible dans la description des fresques de Polygnote à la Leschè de Cnide à Delphes où le Périégète est surpris de voir que l'allure d'Orphée est grecque et que ni son vêtement ni sa coiffe ne sont thraces ${ }^{40}$. Cet étonnement implique que, pour un contemporain de l'historien, Orphée soit thrace. Cette vision déjà présente chez Euripide mais apparemment privilégiée à partir de l'époque hellénistique $^{41}$ se reflète dans le discours que Pausanias prête à un Égyptien.

38. Cinq des treize références à Orphée de la Périégèse renvoient à l'Orphée thrace : II, 30, 1, III, 13, 2, V, 26, 3, IX, 30, 4-12, X, 30, 6-8. Voir Sabbatucci, 1991, p. 7.

39. Pausanias, Périégèse, IX, 27, 2 et X, 7, 2.

40. X, 30, 6-8. L'étonnement de Pausanias peut être partagé puisque, dans l'iconographie de son époque, Orphée porte le plus souvent la tenue phrygienne qui confirme son origine thrace (voir J M. X. Garezou, LIMC, 7, 1, (1994) s. v. «Orpheus », Jesnick, 1997, p. 70-72 et Vieillefon, 2003, p. 49). Il doit cependant être nuancé par le fait que les représentations qui nous sont parvenues appartiennent principalement à l'iconographie romaine. Polygnote a sans doute voulu quant à lui peindre un Orphée grec pour pouvoir mieux l'intégrer à la culture hellénique.

41. Voir T 30-37 Kern ; 929, 1024 I ; 1028, 924 ; 933 ; 921 ; 954 III ; 1051, 1078 II ; 922 ; 556 V-VIII Bernabé. Sur la naissance d'Orphée dans la région de l'Olympe, T 38-41 Kern ; 947, $1010 \mathrm{I}$; $931 \mathrm{I}$; $934 \mathrm{III}$; $974 \mathrm{I}$; $931 \mathrm{I}$, 934 III, 974 I ; 554, 659, 816, 936, 1049 ; 934 V, 937III Bernabé. Les Cicones constituaient un peuple thrace, tout comme les Odryses auxquels est souvent aussi rattaché Orphée. Sur les « trois » Thrace (Mont Hémus, Mont Pangée et Piérie) d'où serait originaire Orphée selon les traditions, Graf, 1987, p. 86-89. 
Celui-ci rapporte qu'Orphée, comme Amphion, avait la terrible capacité du «mage » (mageusai deinon, VI, 20,18). Alors que pour un Grec classique, le pouvoir d'amener le monde naturel à la civilisation semblait relever des prodiges de la mousikê et non de ceux de quelque magie ${ }^{42}$, au II $^{\mathrm{e}}$ siècle après J.-C., début de l'époque des théurges et thaumaturges, Orphée ne chante plus seulement, mais, selon le néologisme d'Apollinaire, « incante ». Rappeler sa nationalité thrace le confirme dans ce rôle. Même si Pausanias refuse de prêter créance au mythe du citharède, fils de la Muse qui enchantait animaux et dieux (IX, 30, 4), il n'en confie pas moins à un Égyptien le soin de définir l'activité d'Orphée en termes contemporains. L'Égypte étant traditionnellement considérée comme le pays où furent instruits tous les «mages » de Pythagore à Jésus en passant par Platon et Moïse ${ }^{43}$, le jugement se veut objectif. Il demeure ambigu en ce qu'une activité définie comme « terrible » effraie plus qu'elle n'est louable. Il reflète néanmoins la pensée des contemporains du Périégète sur Orphée : le citharède est devenu pour eux un «mage».

\section{3) Orphée, «mage » vénérable dans la défense d'Apollonios de Tyane}

La qualification de "mage », peut-être encore équivoque dans le langage courant, acquiert une valeur nettement laudative dans le cadre des procès pour goêteia. La convocation d'Orphée dans la défense d'Apollonios de Tyane fait apparaitre que son application au poète et mystagogue thrace renforce sa connotation positive. Après le rappel du procès intenté au héros de Philostrate, l'examen de lettres attribuées à celui-ci montrera comment la mention d'Orphée dans cette correspondance contribue à la réhabilitation du « mage » - titre qui par là peut même être attribué aux anciens et illustres philosophes.

42. Sabbatucci, 1991, p. 9.

43. Fowden, 1986, p. 79-87 ; Graf, 1996, p. 83. Proclus affirme aussi que Plotin est égyptien (Théologie platonicienne, I, 1, § 2, p. 6, 1. 19). 


\section{3) a. Le procès d'Apollonios}

À la fin du $\mathrm{I}^{\mathrm{er}}$ siècle après J.-C., Apollonios de Tyane aurait été dénoncé par ses détracteurs comme un goês ${ }^{44}$. Lucien, qui lui reproche d'avoir transmis son art de charlatan au maître de l'imposteur Alexandre d'Abonautique, se répand contre ce maître en invectives qui s'appliquent par répercussion à Apollonios (Alexandre, 5). Selon Origène, un biographe qui aurait précédé Philostrate, Méragène, aurait confirmé cette incrimination, disant que des philosophes distingués auraient été séduits par la magie de ce prétendu «mage et philosophe » comme s'ils étaient tombés sur un goês (Cels. VI, 41). Plus tard Dion Cassius, sans doute inspiré par son hostilité à l'encontre de Caracalla qui avait fait construire un temple en l'honneur d'Apollonios reprend la même diffamation : le supposé grand homme n'aurait été rien d'autre selon lui qu'un parfait goês et magos $(77,18)$. Lactance et Eusèbe, pour récuser toute comparaison entre Apollonios et le Christ, ne diront pas autre chose ${ }^{45}$. Associé au mot goês définitivement entaché par la critique de Platon, celui de magos hérite ainsi de la souillure ${ }^{46}$. Tout le plaidoyer de la défense d'Apollonios sera une tentative pour désolidariser les deux termes. Orphée va alors être pris à témoin au sein de cette apologie comme vénérable ancêtre des «mages », conférant à ses lointains successeurs la dignité qui leur était refusée.

44. Sur cette charge et son rapport à celle de mageia, Baur, [1879] 1966, p. 41-45, Meyer, 1917, p. 391-399, Solmsen, 1941, p. 136-174, Smith, 1978, p. 88-90. M. Smith étudie en détail cette charge jusque chez Lactance et Eusèbe dans le traité de ce dernier appelé traditionnellement Contre Philostrate. Sur la figure d'Apollonios magicien, voir aussi Butler, 1948, p. 55-65.

45. Lactance (Institutions divines V, 2, 12-3, 26) et Eusèbe (Contre Philostrate) répondent à Sossianus Hiéroclès, gouverneur de Bithynie au NordOuest de l'Asie Mineure, qui, vers 304, écrit une attaque contre le christianisme en s'appuyant sur une comparaison entre Apollonios et le Christ. Eusèbe tente de convaincre Apollonios de goêteia dans les chapitres 10, 11, 14, 15, 17, 22, 23, 24, 26, 27, 29, 31, 35, 37, 39, 40.

46. Pour une association péjorative des deux termes, hormis les textes de Celse dirigés contre les chrétiens, voir par exemple, avant Platon, Gorgias (DK B 11, 10) et, après Platon, Eschine, 3, 137, Dion Chrysostome 36, 41, les deux textes auxquels nous venons de renvoyer (Dion Cassius 77, 18 et Origène, Cels. VI, 41) et enfin Hérodien 4, 12, 3-4. 
3) b. La défense d'Apollonios. Distinguer le magos du goês : l'exemple d'Orphée

Au début $\mathrm{III}^{\mathrm{e}}$ siècle $^{47}$, Philostrate (170-244/249) entreprend ainsi de décharger Apollonios du crime de goêteia. Il condamne vivement ce type de charlatanerie en affirmant que les dons merveilleux d'Apollonios ${ }^{48}$ dépassent même ceux des «mages » $(V A, \mathrm{I}, 26)$ et sont en réalité le fruit d'une sagesse transcendante qui le rend omniscient. Selon son zélé biographe, Apollonios, qui aurait appris la mageia chez les Mages eux-mêmes (VA I, 2; 27 et 32), devrait néanmoins être distingué d'un magos (VA I, 2). Pourtant, d'après deux lettres transmises indépendamment de la $\mathrm{Vie}^{49}$, celui-ci se serait « en réalité » défendu « lui-même » en revendiquant ce titre, transformant par là le reproche de son rival Euphratès ${ }^{50}$ en compliment. Il lui aurait suffi ainsi d'éviter le terme goês en faveur de magos. Avant de rappeler le sens originel du second, il serait parvenu à en faire un titre de gloire, après l'avoir habilement décerné, entre autres, aux disciples d'Orphée :

"Au même. Tu penses qu'il faut appeler "mages" les philosophes qui suivent Pythagore, et aussi, je suppose, ceux qui suivent Orphée. Mais je pense que même les hommes qui suivent Zeus doivent être appelés "mages", à condition qu'ils soient divins et justes. (Lettre 16, p. 42 Penella, T 85 Kern, 818, 819 I Bernabé). »

47. Soit peut-être avant la mort de Julia Domna en 217 ou même après en 222-235. Sur la datation voir Solmsen, 1941, col. 139 et Bowie, 1978, p. 1670 avec bibliographie sur le sujet.

48. VA VII, 39 VIII, 7, 3. Cf. V, 12 ; Cf. VS 523, 590. Ces dons seraient la compréhension du langage des oiseaux, la connaissance de toutes les langues de l'univers, la pénétration du secret des cœurs, les prophéties, les guérisons.

49. Sur le corpus de lettres attribuées à Apollonios de Tyane, voir l'édition commentée de J. Penella, 1979 et plus particulièrement p. 99-101 sur les lettres 16 et 17 .

50. Euphratès aurait tenté de nuire à Apollonios qui l'aurait vaincu dans l'estime de Vespasien en ternissant sa réputation. Après l'avoir mis aux prises avec les gymnosophistes - épreuve que son adversaire remporta brillamment, il l'aurait calomnié en disant qu'il aurait conspiré avec Nerva contre l'empereur Domitien et aurait tué un jeune homme d'Arcadie pour lire dans ses entrailles la date d'accession au trône de Nerva. Accusation qui, jointe à celle de pratiquer la magie noire, équivalait à une sentence de mort (Butler, 1948, p. 58). 
« Au même. Les Perses appellent "mages" les êtres divins. "Mage" est donc celui qui sert les dieux ou qui est divin par nature. Quant à toi, tu n'es pas un "mage", mais un impie (Lettre 17, p. 44 Penella). »

L'authenticité des lettres d'Apollonios est certes douteuse ${ }^{51}$. S'il est impossible de déterminer avec exactitude la provenance et la date des deux que nous venons de citer, il est néanmoins aisé d'en reconnaître le contexte et de les inscrire dans une polémique caractéristique du $\mathrm{II}^{\mathrm{e}}$ siècle : le procès contre les goêtes. Apulée devra l'affronter à la même époque et sa stratégie apologétique sera presque la même. Seule nous intéresse ici la manière dont Orphée est convoqué pour revaloriser le statut du «mage». Elle constitue alors un indice confirmant la renommée acquise par le poète thrace chez les philosophes dès le $\mathrm{II}^{\mathrm{e}}$ siècle après J.-C.

Pour se défendre, Apollonios (ou le rédacteur anonyme des deux lettres) réhabilite la qualité de magos. Il commence par forcer son correspondant à reconnaître qu'il l'applique lui-même aux philosophes qui perpétuent les doctrines de Pythagore et peut-être d'Orphée. Il renchérit en ajoutant qu'elle doit revenir aussi à ceux qui honorent Zeus, c'est-à-dire aux hommes pieux. Pour confirmer qu'un «mage » est en réalité un être sage (ou philosophe), divin et juste (et non un charlatan ou goês mal intentionné coupable de magie noire), il rappelle l'origine perse du mot et son sens premier : « un serviteur des dieux ${ }^{52}$, et, par extension, « un homme lui-même

51. Certaines sont peut-être de la main d'Apollonios, d'autres sont vraisemblablement forgées, et ce à des époques différentes, dans des esprits différents. Les quatorze qui sont intégralement transmises par la Vie, si elles ne sont pas originelles, sont peut-être composées par Philostrate, à moins que celui-ci ne livre tout simplement ses sources ou ne les révise à sa manière. Mais ni le biographe ne cite toutes les lettres, ni il ne fait allusion à toutes, et son propos semble parfois même contredire celui d'autres lettres qui appartiennent néanmoins au corpus. Celles-ci émanent peut-être alors d'un ou de fonds qu'il ne connaît pas ou dont le contenu ne lui agrée pas, d'un ou de fonds antérieur(s), voire, pour certaines, de sources postérieures, rédigées aux $I V^{\mathrm{e}}$ et $\mathrm{V}^{\mathrm{e}}$ siècles : à cette époque Apollonios s'est acquis un grand nombre d'admirateurs, précisément grâce à la biographie de Philostrate écrite sur les encouragements de Julia Domna peu après l'érection du temple dont nous avons parlé. Sur ce sujet, voir Penella, 1979, p. 23-29.

52. Même définition chez le Ps-Platon, Alcibiade I, 122 a (auquel va renvoyer Apulée), Dion Chrysostome 36, 41 ; 49, 7 ; Diogène Laërce, I, 6. 
divin »-qualifications qui ne peuvent qu'être à l'honneur d'Apollonios et faire défaut à son accusateur. Toute association avec l'art des goêtes est récusée. Même si son rédacteur semble ajouter la proposition avec quelque hésitation, la lettre 16 donne à penser que, dans l'opinion commune, Orphée est déjà considéré comme un être divin, à l'origine d'une forme de sagesse dont les adeptes pourraient alors être désignés sans honte comme des « mages », et qu'il est peut-être lui-même conçu comme le premier d'entre eux.

La provenance du texte étant incertaine, il serait difficile de dire à quel moment cette conviction est généralement admise parmi les païens, si elle ne réapparaissait pas telle quelle chez Apulée. Il est donc permis de penser qu'elle est caractéristique du $\mathrm{II}^{\mathrm{e}}$ siècle. À cette date, en effet, sont déjà ${ }^{53}$ rédigés les Discours sacrés en Vingt-Quatre Rhapsodies attribués à Orphée. Or, les traces d'allégorie stoïcienne, d'influence néo-pythagoricienne voire médio-platonicienne que comportent ces textes reflètent les courants de pensée de l'époque et doivent séduire les esprits cultivés du moment. Il est sûr que si Apollonios ou son défenseur peuvent en appeler au jugement commun sur les philosophes qui se réclament d'Orphée, c'est que l'image «philosophique » de ce dernier n'est plus négative. Quand il met sur le même plan les disciples du poète et les dévots de Zeus par le biais d'une même construction grammaticale avec la préposition apo suivie du génitif, il semble même, par répercussion, ériger Orphée au rang divin ${ }^{54}$. Les philosophes qui suivent ce dernier, du moins, auraient pour lui la même vénération que les desservants d'un dieu. Ainsi associé aux sages de l'Antiquité, Orphée est peut-être

53. Brisson, [1985] 1995, p. 389-420 et [1987] 1995, p. 53 pour une datation des Rhapsodies entre le $\mathrm{I}^{\mathrm{er}}$ siècle après J. -C. et le début du $\mathrm{II}^{\mathrm{e}}$, contra West, 1983 qui situe cette compilation vers 100 avant J. -C. Le débat sur la datation des Rhapsodies est évoqué par A. Bernabé dans le premier tome de son édition des fragments orphiques chez Teubner, 2004, p. 98.

54. Nous avons commenté le texte donné par R. J. Penella qui préfère la leçon tou Dios commune à tous les manuscrits à la leçon tou deinos proposée par Spengel et retenue par les éditions de C. L. Kayser (Teubner, 1870) et de F. C. Conybeare (Harvard University Press, 1948). Si ces derniers avaient néanmoins raison, il faudrait comprendre que, quel que soit le grand homme (ou dieu ?) que l'on suive, du moment que l'on est un homme divin et juste, on mérite le titre de «mage». 
ici dissocié de son image d'auteur de traités magiques et «pseudoscientifiques ${ }^{55}$. Par un tel raisonnement du moins, sa figure de «mage » ne peut plus faire l'objet d'un jugement négatif chez les philosophes. Elle est même revalorisée indirectement par la haute estime d'Apollonios qu'a le rédacteur de la lettre (ou qu'Apollonios a de lui-même) puisqu'il revendique pour lui ce titre dont il vient de prouver la grandeur.

\section{3) c. Le « mage » Orphée aux côtés de Pythagore, Platon et Socrate}

Cette admiration pour Orphée est sanctionnée par un même recours apologétique à son personnage dans une autre lettre non transmise par Philostrate. Là, Apollonios aurait placé le poètephilosophe thrace aux côtés de Pythagore, Platon et Socrate après avoir affirmé, non sans une fierté qui trahit sans doute une défense apocryphe, qu'il aurait lui-même appartenu à la catégorie de ces grands hommes sur lesquels circulent des propos contradictoires ${ }^{56}$. Dieu étant lui aussi soumis à ce mauvais traitement, Apollonios, et Orphée par la même occasion, en tirent a fortiori un titre de gloire supplémentaire :

« S'il y a deux discours qui circulent à mon sujet, et circuleront encore à l'avenir, quoi d'étonnant? Il est nécessaire en effet que des discours contradictoires circulent sur tout individu qui semble supérieur en quelque domaine. Ainsi sur Pythagore, sur Orphée, sur Platon, sur Socrate non seulement furent dites, mais aussi écrites des choses contradictoires ; faudrait-il encore s'en étonner puisque sur Dieu lui-même circulent des propos dissemblables (Lettre 48, § 2, Penella, p. 60-62) ? »

Le rappel du sort d'Orphée, sur lequel circuleraient aussi des propos contradictoires, témoigne, au sujet du poète présenté comme magicien, de l'hésitation dont nous avons fait état. L'auteur de la lettre songe peut-être ici également aux traités que nous qualifierions

55. Nous mettrons toujours cette expression entre guillemets dans la mesure où elle reflète notre point de vue moderne. Il ne s'agit pas ici de juger de la valeur scientifique des écrits attribués à Orphée.

56. On le soupçonnait en effet de ne pas venir en aide à ses compatriotes alors qu'il venait d'offrir généreusement de l'argent à un certain Diotimus de Tyane qui n'était ni son parent ni son ami. Voir Penella, 1979, p. 115. 
de «pseudo-scientifiques » attribués à Orphée, voire à l'utilisation du nom de celui-ci par les magiciens, qui contribuent à une réputation contrastée du personnage selon les milieux. Pareille réputation s'attache visiblement à Apollonios, considéré tantôt comme un goês et faiseur de tours, tantôt comme un sage. Il importe alors de dissocier le magos du goês et de placer Orphée uniquement du côté des philosophes, qui, en raison de l'évolution des cadres de pensée de l'époque, peuvent être appelés magoi au sens de «desservants des dieux dont les ancêtres spirituels sont d'antiques barbares venus d'Orient ». L'invocation du poète thrace au sein d'une apologie suppose du moins que l'hésitation à son sujet soit levée à l'époque d'Apollonios (ou du moins de son avocat inconnu). Cette hypothèse est ici étayée par la comparaison avec les penseurs dont la réputation n'a plus à être défendue et avec Dieu lui-même ${ }^{57}$.

L'appartenance d'Orphée à ce cortège suggère ainsi que le poète est reconnu comme un sage par les philosophes. Au sein du plaidoyer, son évocation contribue à revaloriser le statut du magos. Par répercussion, sa propre image de «mage » est réhabilitée, à condition du moins, dans ce cadre philosophique, qu'elle reste fidèle à la définition du terme pris au sens de «sage, desservant des dieux, dont les origines spirituelles remontent aux vénérables Barbares », et ici, en l'occurrence, aux Thraces.

Ainsi, dès le II ${ }^{\mathrm{e}}$ siècle après J.-C., la figure d'Orphée «mage » fait l'objet d'estime sinon de vénération, puisqu'il est possible de la prendre à témoin afin de se défendre contre l'accusation de goêteia et de la placer aux côtés des anciens philosophes. Il suffit pour cela de rendre au terme magos son sens originel de «digne desservant des dieux» et de rappeler son origine perse. L'argumentation d'Apollonios ou de son défenseur apocryphe se révélera à ce point convaincante que l'accusé se verra lui-même honoré comme un

57. Pour défendre Apollonios contre l'accusation d'être un « mage » (là où celui-ci aurait plutôt revendiqué le titre en le délestant de sa charge négative), Philostrate l'assimile semblablement à Empédocle, Pythagore, Démocrite, Platon, Socrate et Anaxagore qui, pour avoir fréquenté les Mages et les Égyptiens ou pour avoir été capables de prédire l'avenir, auraient eux aussi pu être accusés de mageia (VA I, 2). 
dieu auprès d'Orphée et même du Christ dans le fameux lararium d'Alexandre Sévère, le fils de son admiratrice Julia Domna ${ }^{58}$.

\section{4) Orphée, «mage » philosophe chez Apulée}

Apulée se fonde sur cette renommée d'Orphée «mage » déjà accréditée lorsque, dans le De Magia, il use de la même stratégie apologétique qu'Apollonios. Après avoir redéfini la magie comme philosophie ou voie menant véritablement au divin, il prend à son tour Orphée comme exemple afin de confirmer son propos et de sceller sa défense. Son raisonnement diffère peut-être cependant de celui des avocats d'Apollonios sur un point : comme il est lui-même intéressé par les sciences naturelles, Apulée fait sans doute aussi allusion à l'Orphée auteur de traités «pseudo-scientifiques ». Par répercussion, ce dernier voit sa figure de magicien complètement réhabilitée, même si, ici encore, cette revalorisation passe par le recours la définition du magos comme « sage et desservant des dieux, venu d'Orient à l'origine ». Le propos d'Apulée une fois examiné, il sera possible d'évoquer l'expansion de cette nouvelle aura qui entoure le «mage » thrace.

Lors du procès pour magie (réel ou imaginaire ${ }^{59}$ ) qu'on lui aurait intenté vers 156-161 après J.-C., Apulée aurait répliqué en retournant lui aussi l'accusation en compliment. Revendiquant le titre de philosophe et d'homme pieux, il aurait affirmé que le terme de «mage » répondait précisément à cette définition ${ }^{60}$. Pour le prouver, dans la version écrite de son discours, il commence par rappeler la définition perse du substantif magus, qu'il donne comme synonyme du latin sacerdos, c'est-à-dire «individu qui connaît,

58. Histoire Auguste, Alexandre Sévère, 29, 2 (texte dont l'information demeure douteuse et qui relève peut-être de la propagande).

59. Sur ce sujet, Graf, 1996, p. 61-82, Hammerstaedt, Habermehl, Lamberti, Ritter, Schenk, 2002. Sur l'authenticité de ce procès et la défense d'Apulée comme texte réellement prononcé, retravaillé pour la publication ou seulement inventé comme occasion de se présenter, Hammerstaedt, Habermehl, Lamberti, Ritter, Schenk, 2002, p. 39-46 (P. Schenk).

60. De Magia, 1, 3; 3, 5. Sur la définition de la « magie » par Apulée dans cette apologie, voir Abt, 1908, p. 106-134. 
sait et maîtrise parfaitement les lois relatives aux cérémonies, les règles concernant les sacrifices, les normes religieuses » (25, 8-9). Pour appuyer ses dires, il cite un texte qu'il croit être de Platon évoquant l'éducation royale en Perse où l'homme le plus sage est chargé d'enseigner au prince, outre l'art de gouverner, la mageia, c'est-à-dire la «manière de servir les dieux ${ }^{61}$. La magie serait donc un art honorable, idée confirmée par une autre citation de Platon qui rappelle que les incantations du thrace Zalmoxis sont de « beaux discours » $(26,4)$. Apulée qui, à dessein, ne prête aucune attention à la suite du Charmide où Socrate conclut que, si le jeune homme est vraiment sage, il n'a nul besoin d'incantations (158 b), détourne la pensée du philosophe ${ }^{62}$ pour revendiquer l'usage de telles prétendues «bonnes paroles» $(26,5)$. Avant de donner une définition du «mage » qui soit entièrement à son honneur, il rappelle celle de ses adversaires. Elle correspond au sens commun du mot $(26,6)$ qui renvoie à l'exercice de la magie noire $(47,3)$ et semble correspondre à la définition de la goêteia que Platon donne dans les Lois (X, 909 b 6-d 1). Le «mage» se caractériserait en effet selon elle par sa prétention à communiquer directement avec les dieux pour obtenir d'eux toutes choses incroyables grâce à la force des incantations ${ }^{63}$. Après s'être délesté de cette charge qui accable l'individu nommé goês en grec, Apulée explique que ce sont les ignorants qui, en réalité croient blâmer certains philosophes en les qualifiant de «mages » $(27,1)$. Ils traitent en effet les uns d'irreligiosi sous prétexte que leur recherche des explications mécanistes les conduit à nier l'existence des dieux ${ }^{64}$; les autres de magi.

Orphée est alors pris comme exemple dans la définition de ce dernier terme et contribue ainsi à sa réhabilitation :

61. Alcibiade, I, 121 e-122 a, De Magia, 25, 11. Apollonios aurait renvoyé à la même définition.

62. Abt, 1908, p. 116 écrit : «sein Beweis ist lediglich aufs Verblüffen berechnet. »

63. 26, 6. Voir Abt, 1908, p. 108-113 et 118-119, Habermehl, 2002, p. 290291. Selon Apulée, le vrai «mage » sert ainsi les dieux de manière désintéressée, tandis que le « magicien » ou sorcier tente de les utiliser à des fins personnelles.

64. Sont ainsi visés Anaxagore, Leucippe, Démocrite et Épicure, De Magia, 27, 1. 
« Ceux qui cherchent avec trop de curiosité la providence du monde et fréquentent les dieux avec trop d'empressement, ceux-là, on les nomme couramment "mages", comme s'ils savaient aussi faire des choses dont ils savaient comment elles se produisent ${ }^{65}$, de même qu'il y eut autrefois Épiménide, Orphée, Pythagore et Ostanès ; furent ensuite de même suspectés les Catharmes d'Empédocle, le démon de Socrate et le Bien de Platon (27, 2-3 ; T 85 Kern, 818, 819 I Bernabé). »

Ce passage peut certes être lu de la même façon que la lettre 48 d'Apollonios. Il prouve en effet que la renommée d'Orphée est assurée, puisqu'elle sert la défense du «mage». En retour, cette apologie étaie l'image positive du chantre conçu comme «mage » - réhabilitation qui dépend de la définition donnée à ce terme. Or, c'est là que l'argumentation diffère légèrement de celle des apologistes d'Apollonios : le rhéteur africain inclut dans le rôle du « mage » l'activité « pseudo-scientifique » à laquelle on lui reproche de s'adonner. Dans ce cadre, l'Orphée du $\mathrm{II}^{\mathrm{e}}$ siècle peut servir d'adjuvant idéal.

Apulée définit en effet le «mage » comme un être doué d'une ardente aspiration à connaître la nature ou à entrer en contact avec le divin dont les profanes ne perçoivent pas la grandeur et qu'ils jugent suspecte. Si le rhéteur ne souhaite pas être qualifié par un terme, qui, dans l'esprit de ses contradicteurs, demeure synonyme du grec goês, il refuse néanmoins leur critique. L'étude de la nature n'est pas l'athéisme et la recherche zélée du divin n'est pas magie au sens de « sorcellerie », art qui prétendrait produire les phénomènes dont il comprend les causes. Toutes deux relèvent au contraire de la médecine et de la philosophie. Apulée réunit ici sans doute pour sa défense les deux images d'Orphée perçues comme contradictoires ${ }^{66}$. Son raisonnement sous-jacent à l'égard du poète thrace serait le suivant. En vertu des critères philosophiques de l'époque, Orphée est devenu un personnage digne de figurer aux côtés des sages les plus vénérables. Preuve en serait l'apparition des Rhapsodies mises sous son nom. Or, des traités sur les plantes et les pierres, par exemple,

65. Formulation qui décrit l'activité proprement magique et résume le procès intenté aux prétendus magiciens : l'étude de la nature supposerait de leur part le désir suspect de reproduire les œuvres divines.

66. Sur cette image contradictoire, Bernabé, 1998 et Hernández 2003. 
lui sont progressivement ${ }^{67}$ attribués. Apulée mentionne lui-même certains remèdes que le poète et mystagogue thrace aurait découverts $(30,11)$. Cette activité que nous qualifierions de «pseudo-scientifique » serait donc digne de respect. Elle ne serait pas goêteia, mais éventuellement mageia prise au sens de « science » et de « philosophie » et dont les origines remonteraient aux sages venus d'Orient (ou ici de Thrace). Les auteurs d'ouvrages attribués à Orphée usent du moins vraisemblablement de ce raisonnement. Apulée semble ici les confirmer ${ }^{68}$. Son argumentation est alors parfaite par un retour à la majeure : Orphée peut être compté parmi les philosophes.

Le rhéteur africain a en effet l'habileté de mettre la discipline royale, la philosophie, sur le banc des accusés. La définition qu'il donne des «mages » est volontairement provocatrice. Il inscrit au nombre de ceux que ses détracteurs nomment ainsi le Perse Ostanès («mage » au sens originel du terme), les philosophes « de la nature» à la piété célèbre ou fondateurs de mouvements religieux comme Épiménide, Empédocle et Pythagore, ou encore Socrate et Platon en faisant allusion au respect et à la pensée du divin de ces derniers. Cela revient à invalider définitivement le mépris accordé à la qualification de magos. La présence d'Orphée dans ce cortège prouve ici encore que les contemporains d'Apulée tiennent le poète thrace en haute estime. Par répercussion, sa figure de «mage » et, cette fois, de magicien doivent cesser de faire l'objet d'invectives. Pouvoir se réclamer de lui et de ses confrères, comme l'aurait fait Apollonios avant Apulée, devient un honneur pour l'accusé qui ne tarde pas à s'en vanter : « je me félicite donc d'être, moi aussi, compté parmi des hommes si nombreux et si grands $(27,4)$. » La magie d'Apulée est philosophie.

La stratégie apologétique appliquée ici révèle une fois encore le crédit que les contemporains de l'accusé accordent nécessairement

67. Ces traités appartiennent pour la plupart à l'époque tardive, mais se multiplient dès le $\mathrm{II}^{\mathrm{e}}$ siècle après J.-C. (voir Hernández, 2003, p. 66-73, 2006 a, 2006 b, p. 275-342).

68. A. Abt (1908) met bien en évidence que la définition du «mage» donnée par Apulée est exactement celle revendiquée par les auteurs des papyri magiques. 
à Orphée dont la figure de «mage » et plus généralement de magicien se voit alors implicitement réhabilitée au cours d'un tel procès. Orphée apparaît en effet dans un brillant cortège composé des anciens philosophes et sa «magie » est, comme celle d'Apulée, médecine, philosophie ou théologie, mais non plus « sorcellerie » (goêteia) ni impiété.

\section{Conclusion. L'aura du « mage » Orphée}

à partir du II siècle après J.-C.

À l'occasion des procès pour «magie», le titre de «mage» devient laudatif sous la plume habile de la défense. Il est pour cela ramené à son sens originel de « sage, desservant des dieux » et son application à Orphée contribue à sa réhabilitation - preuve que la réputation de ce dernier est devenue positive chez les philosophes de l'époque. Pour s'en convaincre, il suffit de voir qu'Orphée peut être placé aux côtés de Pythagore et Platon sans que cela choque le lecteur. Par répercussion non seulement la figure d'Orphée «mage » est revalorisée, mais même celle du magicien. La retenue de Pausanias est abandonnée. Dans ce contexte, il n'est même plus déshonorant pour le poète thrace de se voir attribuer, à côté de réflexions médicales voire d'un ouvrage sur les traitements par les plantes et les herbes ${ }^{69}$, un traité de magie sur l'utilisation des pierres, les Lithiques, dont la composition remonte peut-être précisément au $\mathrm{II}^{\mathrm{e}}$ siècle $^{70}$. L'évolution de la philosophie, qui se conçoit de plus en plus comme une théologie, privilégie les contacts avec le divin et exalte les sages et antiques « Barbares », joue un rôle essentiel dans le nouveau prestige du thrace Orphée, qui devient l'auteur des Rhapsodies, vers peut-être

69. F 319-331 Kern, 785, 786 ; 787, 792 ; 796 ; 818 II ; 788 ; 790 ; 791 ; 797 ; 793 ; $794 ; 798$; 799 Bernabé. Nous avons vu que Pline, qui voit en Orphée un médecin (Histoire naturelle XXX, 7), lui attribue aussi des connaissances sur les herbes $(\mathrm{XXV}, 12)$ ou sur les potions d'amour $(\mathrm{XX}, 32)$. Apulée rappelle aussi toujours pour se défendre que ce que ses accusateurs appellent poisons, ce sont en réalité des herbes telles qu'on en trouve beaucoup chez Orphée (De Magia, XXX, 35). Sur Orphée « médecin », voir Coman, 1938, p. 159-160.

70. Schamp, 1981, Halleux, Schamp, 1985, Brisson, 1993, p. 182, Bernabé, 1998, p. 171, Hernández, 2003, p. 69-70, 2006 b, p. 318-341. 
composés par des philosophes ou du moins interprétés par eux de manière privilégiée. Elle contribue même sans doute aussi à la réhabilitation de sa figure de magicien, laquelle est du moins scellée par les prétentions des accusés pour magie au titre de philosophes. Ainsi, à partir du $\mathrm{II}^{\mathrm{e}}$ siècle, sur la scène littéraire, l'image désastreuse du goês cède la place à celle, vénérable, du « mage ». Plus tard, même celle du sophiste sera réhabilitée ${ }^{71}$. Philostrate, qui décharge le « mage » Apollonios du soupçon de goêteia, vantera l'art des anciens et nouveaux sophistes en disant que leurs discours sont des ôidai et qu'ils savent charmer leur auditoire à la manière d'Orphée et Thamyris ${ }^{72}$. Au XII ${ }^{\mathrm{e}}$ siècle byzantin enfin, il n'y aura plus d'hésitation à louer Orphée comme le fondateur de la magie. Jean Tzetzes pourra affirmer dans son Commentaire à l'Iliade qu'Homère s'était rendu en Égypte pour apprendre auprès du chantre thrace cet art « grandement digne d'éloges ${ }^{73}$.

La qualification de magicien attribuée à Orphée, négative dans la période classique et les textes qui restent attachés à l'esprit de ce temps, souvent associée au rappel de la nationalité thrace et donc barbare, devient source d'éloges dans certains textes à partir du II ${ }^{\mathrm{e}}$ siècle après J.-C. Dans le premier cas, la condamnation s'appuie sur le souvenir de la célèbre critique de Platon (Strabon) ou sur un jugement populaire défavorable au chantre qui peut lui aussi remonter à la critique platonicienne (Pline). Dans le second, l'excellente

71. Clément d'Alexandrie par exemple utilise le substantif sophistês de manière certes ambiguë, mais vraisemblablement péjorative pour désigner Orphée dans le Protreptique, I, 1, 1. Sur ce sujet, voir le premier tome de notre thèse de doctorat à paraître et l'article de J.-M. Roessli, 2002, p. 506, n. 6.

72. VS I, 483 sur Prodicos et 520 sur Scopélien. Lettres p. 364. Protagoras, dont le père était thrace, aurait d'ailleurs lui aussi étudié en Perse chez les magoi (VS 494, 10)?

73. Commentaire à l'Iliade, 17, 19. T 86 Kern, 824 II Bernabé. Un autre passage de Jean Tzetzes explique cette admiration. Selon lui, Orphée, par sa musique, aurait éveillé Eurydice mordue par le serpent (Historiarum Variarum : Chiliades, II, 54, 1. 843-857). Voir Friedman, [1970] tr. fr. 1999, p. 183 et Vicari, 1982, p. 77. 
renommée du poète, due entre autres à un changement dans la manière de concevoir la philosophie, suggère de convoquer ce dernier dans la défense des «mages ». Par ce processus, il acquiert le titre, lui confère en retour ses lettres de noblesse et voit par répercussion réhabilitée sa propre figure de magicien. Il est alors digne de figurer aux côtés de Pythagore, Socrate et de son plus grand ennemi : Platon lui-même ! Ainsi, lorsqu'à la même époque, Clément d'Alexandrie reprend les termes du procès de la République et des Lois à l'encontre du goês Orphée, il fait volontairement fi de la réputation du poète thrace chez certains de ses contemporains et peut-être condisciples (Apulée pouvant être considéré comme philosophe médio-platonicien) afin de défendre un autre accusé pour goêteia, le Christ lui-même ${ }^{74 *}$.

7, rue du Quatorze Juillet

F-28000 Chartres

\section{BIBLIOGRAPHIE}

Авт, Adam, 1908, Die Apologie des Apuleius von Madaura und die antike Zauberei, in Religionsgeschichtliche Versuche und Vorarbeiten, 4, 2, p. 75345.

BAUR, Ferdinand Christian, [1876] 1966, Drei Abhandlungen zur Geschichte der alten Philosophie und ihres Verhältnisses zum Christentum, « Apollonius von Tyana und Christus », Zeller, Leipzig.

Bernabé, Alberto, -1998, «La Palabra de Orfeo: Religión y magia », in Er, Revista de Filosofía, Documentos 1998, p. 157-172.

- 2001, «Un "resumen de historia del orfismo" en Estrabón VII fr. 18 », in Actas Sociedad española de Estudios Clásicos, Madrid, p. 59-66.

- 2003, «Las Ephesia Grammata. Génesis de una fórmula mágica», in Revista internacional de investigación sobre magia y astrología antiguas 2003 , 3, p. 5-28.

74. Sur ce sujet, voir Smith, 1978 et les critiques de cet ouvrage résumées dans Ritter, 2002, p. 317.

* Je remercie Jean-Michel Roessli pour sa relecture du texte, ses corrections et ses généreux conseils bibliographiques, ainsi qu'Alberto Bernabé qui m'a également donné des indications bibliographiques. 
- 2006, «Magoi en el Papiro de Derveni: ¿Magos Persas, charlatanes u oficiantes órficos? », in CALDERÓN, Esteban, MorALES, Alicia, VALVERDE, Mariano (éd.), Koinòs lógos, « Homenaje al profesor José García López », Universidad de Murcia, Murcia, p. 99-109.

Betegh, Gábor, 2004, The Derveni Papyrus, « Cosmology, theology and interpretation », Cambridge University Press, Cambridge.

Bidez, Jean, Cumont, Franz, 1938, Les Mages hellénisés, «Zoroastre, Ostanès et Hystaspe d'après la tradition grecque », Les Belles Lettres, Paris.

Boyancé, Pierre, [1936] 1972², Le Culte des Muses chez les Philosophes grecs, «Étude d'histoire et de psychologie religieuse », De Boccard, («Bibliothèque des écoles françaises d'Athènes et de Rome » 141), Paris.

BowIE, Ewen, 1978, « Apollonios of Tyana: Tradition and Reality », in Aufstieg und Niedergang der römischen Welt II, 16, 2, p. 1652-1699.

BREMMER, Jan, 1999, « The Birth of the term "magic" », in Zeitschrift für Papyrologie und Epigraphik 126, p. 1-12.

Brisson, Luc, -1985, «Les théogonies orphiques et le Papyrus de Derveni : notes critiques », in Revue de l'histoire des religions 202, p. 389-420.

- 1987, «Proclus et l'orphisme », in Proclus. Lecteur et interprète des Anciens, Colloques internationaux du CNRS, CNRS, Paris, p. 43-103. Réimprimé dans Brisson, L., Orphée et l'Orphisme dans l'Antiquité gréco-romaine, Ashgate («Variorum »), Aldershot, 1995.

- 1993, Postface à Orphée, Poèmes magiques et cosmologiques, Les Belles Lettres (« Aux sources de la tradition»), Paris, p. 153-190.

- 2000, «L'incantation de Zalmoxis dans le Charmide (156d-157c) », in Brisson, Luc, Robinson, Thomas M., (éd.), Plato, Euthydemus, Lysis, Charmides, "Proceedings of the V Platonicum, Selected papers », Academia Verlag, Sankt Augustin, p. 278-286.

BURKERT, Walter, 1962, «Goês. Zum griechischen Schamanentum », in Rheinisches Museum 105, p. 36-55.

Butler, Eliza Marian, 1948, The myth of the Magus, Cambridge University Press, Cambridge ; The Macmillan Company, New York.

CASAdesús Bordoy, Francesc, 1998, « La crítica platónica de la magia », in Peláez, Jesus (éd.), El Dios que Hechiza y encanta, « Magia y Astrología en el Mundo Clásico y Helenístico », Actas del I Congreso Nacional Córdoba 1998, Ediciones El Almendro Córdoba, Madrid, p. 191-201.

- 2001, «Análisis de la figura del mago en ambientes órficos », in Actas del X congreso de la sociedad española de Estudios Clásicos, Vol. III, Sociedad española de Estudios Clásicos, Madrid, p. 75-82.

Coman, Jean, 1938, « Orphée, civilisateur de l'humanité », in Zalmoxis 1, p. $130-176$. 
DÖRRIE, Heinrich, 1972, « Die Wertung der Barbaren im Urteil der Griechen. Knechtsnaturen? Oder Bewahrer und Künder heilbringender Weisheit? », in Stiehl, Ruth, Lehmann, Gustav Adolf, Antike und Universalgeschichte, «Festschrift Hans Erich Stier zum 70. Geburtstag am 25. Mai $1972 »$, Aschendorffsche Verlagsbuchhandlung, («Fontes et commentationes » suppl. 1), p. 146-175.

FARAONE, Christopher, OBbink, Dirk (éd.), 1991, Magika Hiera, « Ancient Greek Magic and Religion », Oxford University Press, New York, Oxford.

Fowden, Garth, 1986, The Egyptian Hermes. A Historical Approach to the Late Pagan Mind, Cambridge University Press, Cambridge.

FrIEDMAN, John Block, [1970] 1999, Orpheus in the Middle Ages, Harvard University Press, Cambridge (Mass.). Trad. fr. Orphée au Moyen Âge, par Jean-Michel Roessli, avec le concours de V. Cordonier et F.-X. Putallaz, Cerf («Vestigia » $\mathrm{n}^{\circ} 25$ ), Paris-Fribourg, 1999, avec postface de JeanMichel Roessli.

GraF, Fritz, -1987, « Orpheus: A poet among Men », in Bremmer, Jan (éd.), Interpretations of Greek Mythology, Croom Helm, London and Sidney, p. 80-106.

- 1996, Gottesnähe und Schadenzauber, «Die Magie in der griechischrömischen Antike », C. H. Beck, München.

HalleuX, Robert, Schamp, Jacques, 1985, Les Lapidaires grecs, Les Belles Lettres («CUF»), Paris.

Hammerstaedt, Jürgen, HabermeHl, Peter, LAmberti, Francesca, RitTer, Adolf Martin, Schenk, Peter, 2002, Apuleius, De Magia, (Eingeleitet, übersetzt und mit interpretierenden Essays versehen von) Wissenschaftliche Buchgesellschaft (« Sapere »), Darmstadt, 2002.

HERnÁndez, Martín Raquel, -2003, « La relación de Orfeo con la magia a través de los testimonios literarios », in Revista internacional de investigación sobre magia y astrología antiguas 2003, 3, p. 55-74.

- 2005, «Orfeo, el Orfismo y la magia en los S. V y IV a. C. », in Actas del XI congreso de la sociedad española de Estudios Clásicos, Sociedad española de Estudios Clásicos, Madrid, p. 375-383.

- 2006 a, « Orfeo científico », in Estudios Clásicos 129, p. 111-119.

- 2006 b, El Orfismo y la magia, Universidad Complutense de Madrid (thèse disponible sur Internet à l'adresse suivante : www. ucm.es/BUCM/tesis/ fll/ucm-t29556.pdf).

Honigmann, Ernst, 1931, article « Strabon », in Pauly's Realencyclopädie der classischen Altertumswissenschaft (2. Reihe) IV, A, 1, col. 76-155.

JESNICK, Ilona Julia, 1997, The Image of Orpheus in Roman Mosaic, « An Exploration of the Figure of Orpheus in Graeco-Roman Art and Culture with Special Reference to its Expression in the Medium of Mosaic in Late Antiquity », BAR International Series 671, Oxford. 
JORDAN, David, 1992, «The Inscribed Lead Tablet from Phalasarna », in Zeitschrift für Papyrologie und Epigraphik 94, p. 191-194.

- 2000 a, «Three texts from Lokroi Epizephyrioi », in Zeitschrift für Papyrologie und Epigraphik 130, p. 95-103.

- 2000 b, « Ephesia grammata at Himera », in Zeitschrift für Papyrologie und Epigraphik 130, p. 104-107.

Jourdan, Fabienne, 2003, Le Papyrus de Derveni, Les Belles Lettres, («Vérité des mythes »), Paris.

JÜTHNER, Julius, 1950, article «Barbar » in Reallexicon für Antike und Christentum 1, col. 1173-1176.

Kollmann, Bernd, 1996, Jesus und die Christen als Wundertäter, Vandenhoeck und Ruprecht, Göttingen.

KoTANSKY, Roy, 1991, « Incantations and Prayers for Salvation on Inscribed Greek Amulets », in FARAOne, Ch., OBbink, D. (éd.), p. 107-137.

LÉVY, Edmonds, 1984, « Naissance du concept de barbare », in Ktèma, 9, p. 5-14.

Meyer, Eduard, 1917, Apollonios von Tyana und die Biographie des Philostrates, in Hermès 52, p. 317-424 (repris dans Kleine Schriften, 1924, II, p. 131-191).

OPELT, Ilona, Speyer, Wolfgang, 1967, « Barbar. Nachträge zum Reallexikon für Antike und Christentum », in Jahrbuch für Antike und Christentum, p. 251-290.

Penella, Robert, 1979, The Letters of Apollonius of Tyana, « a Critical Text with Prolegomena, Translation and Commentary », Brill (« Mnemosyne », Bibliotheca Classica Batava), Leyden.

REverdin, Olivier, 1961, « Crise spirituelle et évasion », in Schwabl, Hans et al., 1961, p. 85-120.

RITTER, Adolf Martin, 2002, « Magie im frühen Christentum », in HAMMERStaedt, J., Habermehl, P., LAmberti, F., Ritter, A. M., Schenk, P., 2002, p. 314-330.

RoESSLI, Jean-Michel, 2002, « Convergence et divergence dans l'interprétation du mythe d'Orphée, de Clément d'Alexandrie à Eusèbe de Césarée », in Revue de l'histoire des religions 219, p. 503-513.

Romilly, Jacqueline, 1974, Magic and Rhetoric in Ancient Greece, Harvard University Press («The Carl Newell Jackson Lectures »), Cambridge (Mass.).

SabBatucci, Dario, 1991, «Orfeo secondo Pausania » in Borgeaud, Philippe (éd.), Orphisme et Orphée, "En l'honneur de Jean Rudhardt », Droz («Recherches et Rencontres »), Genève, p. 7-11.

ScHAMP, Jacques, 1981, « Entre Hermès et Zoroastre. Observations sur la datation traditionnelle du Lapidaire orphique », in L'Antiquité classique 50, p. 721-730. 
Schwabl, Hans et al., 1961, Grecs et Barbares, « Six exposés et discussions », in Entretiens sur l'Antiquité classique Tome VIII, Fondation Hardt, Vandœuvres-Genève.

SiRINELLI, Jean, 1993, Les Enfants d'Alexandre, « La littérature et la pensée grecques de 334 av. J.-C. à 529 après J.-C. », Fayard, Paris.

SMith, Morton, 1978, Jesus the Magician, Victor Gollancz, London.

Solmsen, Friedrich, 1941, article «Philostratos », in Pauly's Realencyclopädie der classischen Altertumwissenschaft 20, 1, p. 136-174 (repris dans Kleine Schriften, 1968, II, p. 97-116).

TüleK, Füsun, 1998, Efsuncu Orpheus/Orpheus the Magician, «The Transition of Orpheus Theme between Paganism and Christianity in Late Roman Early Byzantine Mosaics », Arkeoloji ve sanat Yayinlari, Istanbul.

VICARI, Patricia, 1982, «Orpheus among the Christians », in WARDEN, John (éd.), Orpheus, «The Metamorphoses of a Myth», University of Toronto Press, Toronto-Buffalo-London, p. 63-84.

VIEILLEFOn, Laurence, 2003, La Figure d'Orphée dans l'Antiquité tardive, «Les mutations d'un mythe : du héros païen au chantre chrétien », De Boccard (De l'archéologie à l'histoire), Paris.

West, Martin, 1983, The Orphic Poems, Clarendon Press, Oxford. 